\title{
Metal Flux from Dissolution of Iron Oxide Grain Coatings in Sandstones
}

\author{
J. Parnell $\mathbb{D},{ }^{1}$ X. Wang $\mathbb{D},{ }^{2}$ A. Raab $\mathbb{D}^{2},{ }^{2}$. Feldmann $\mathbb{D}^{2,3}$ C. Brolly $\mathbb{D}^{1},{ }^{1}$ R. Michie $\mathbb{D}^{1}{ }^{1}$ \\ and J. Armstrong ${ }^{1}$ \\ ${ }^{1}$ School of Geosciences, University of Aberdeen, Aberdeen AB24 3UE, UK \\ ${ }^{2}$ Trace Element Speciation Laboratory (TESLA), Department of Chemistry, University of Aberdeen, Aberdeen AB24 $3 U E$, UK \\ ${ }^{3}$ Environmental Analytical Chemistry, Institute of Chemistry, University of Graz, Graz, Austria
}

Correspondence should be addressed to J. Parnell; j.parnell@abdn.ac.uk

Received 28 January 2021; Revised 11 March 2021; Accepted 20 April 2021; Published 18 May 2021

Academic Editor: Guo Xiang Chi

Copyright @ 2021 J. Parnell et al. This is an open access article distributed under the Creative Commons Attribution License, which permits unrestricted use, distribution, and reproduction in any medium, provided the original work is properly cited.

\begin{abstract}
Iron oxide grain coatings in red sandstones contain trace metals that are released upon dissolution of the coatings. Analyses by ICPMS following acid leaching of the grain coatings show that the dissolved metals can constitute an ore-forming fluid, as hypothesized in models for sandstone-hosted ore deposits. Median compositions of 37 samples, mostly of Triassic to Devonian age, from across Britain and Ireland are $6.3 \mathrm{ppm}$ copper, $2.4 \mathrm{ppm}$ cobalt, $10.1 \mathrm{ppm}$ vanadium, and $0.3 \mathrm{ppm}$ uranium. These contents at the basin scale are adequate to form the observed range of ore deposits in red beds. The migration of hydrocarbons or brines can cause the dissolution of grain coatings and contributes to controlling the distribution of ore deposits. Future measurements should test red beds derived from uplifted, mineralized plate margins, in which sandstones may be preloaded with ore metals.
\end{abstract}

\section{Introduction}

The mineralization of sedimentary basins has made an important contribution to the planet's resources of metals [1]. Mineralization in basins requires processes that concentrate metals beyond their normal abundance by several orders of magnitude. Examples include the concentration of redox-sensitive elements by reduction sites, such as black shales, and the release of metals from clays during diagenesis. In continental basins, one of the most widespread of processes is the formation of iron oxide coatings around sand grains during early diagenesis [2]. The iron oxide derives from the alteration of mineralogically immature grains such as ferromagnesian minerals. The coatings are what make many sandstones red, when the iron oxide becomes the mineral haematite. Iron oxides are particularly significant to diagenesis because of their capacity to adsorb a wide variety of trace elements from groundwaters [3]. As diagenesis proceeds, and the volume of secondary iron oxides increases, the proportion of trace elements resident in the oxides also increases. However, the grain coatings are also susceptible to removal by dissolution into acidic pore fluids, including oilfield brines [4]. For example, dissolution is evident from the bleaching of red beds following hydrocarbon migration (e.g., [5]) and from $\mathrm{CO}_{2}$-rich brines [6, 7], but these fluids are not always evident. The combination of trace metal uptake and dissolution is a potential pathway to produce a mineralizing fluid [8-12]. This possibility has been invoked to explain moderate- to large-scale ore deposits in sandstones, such as copper and silver in Kazakhstan [13, 14], copper and silver in Iran [15], uranium in Australia [16], copper in Argentina [17, 18], vanadium in Argentina [19], copper in Arctic Canada [20], copper in Newfoundland [21], lead-zinc in China [22], and uranium in China [23]. The occurrence of red sandstones does not, however, necessarily explain mineralization, as other sources such as underlying basement may contribute more metal in certain hydrological regimes [24].

The high abundance of red beds, in space and time [25], implicates their potential importance to the mineralization of continental basins. Traces of mineralization occur in red beds 


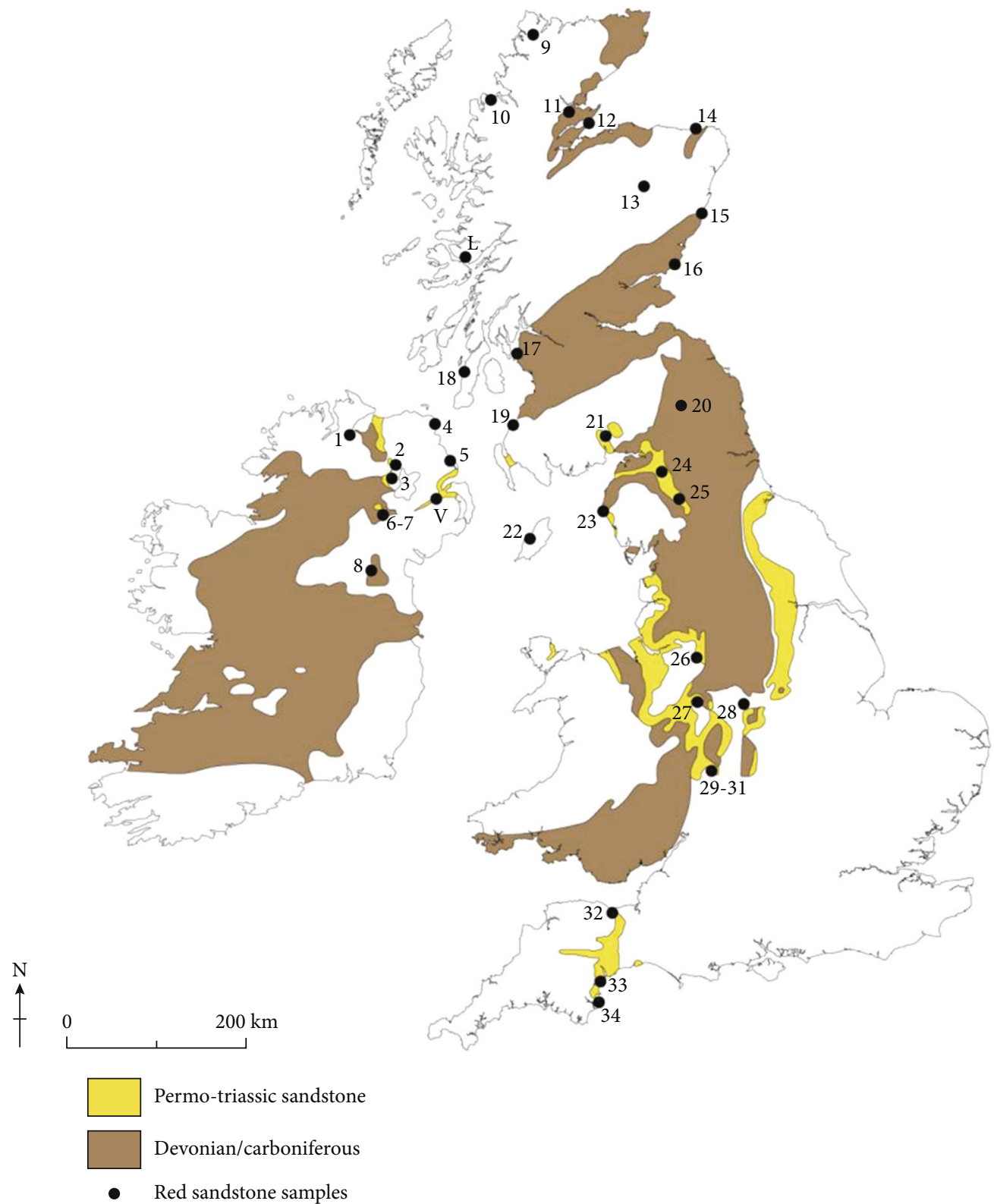

Figure 1: Locations for red sandstone samples, Britain and Ireland. Numbered localities were sampled for red sandstones (Table 1). L: Lochaline quartz sandstone procedural blank. V: Lagan Valley borehole 4B sandstone used for LA-ICP-MS map.

dated at least back to the Mesoproterozoic [26]. However, there are only very limited data for the trace element compositions of the iron oxide coatings that are central to the process. This study reports the compositions of grain coatings from numerous different localities and ages.

The objectives are specifically:

(i) Measurement of a data base of compositions of grain coatings. Data were collected for vanadium $(\mathrm{V})$, cobalt $(\mathrm{Co})$, copper $(\mathrm{Cu})$, selenium $(\mathrm{Se})$, uranium $(\mathrm{U})$, arsenic $(\mathrm{As})$, and lead $(\mathrm{Pb})$ in iron- $(\mathrm{Fe}-)$ rich grain coatings. These elements represent those which commonly occur in red bed ore deposits (ii) Assessment of whether the composition of grain coatings is related to their relative abundance, i.e., quantity of iron oxide

(iii) Assessing the typical range of metal fluxes that might be expected from their dissolution

\section{Methods}

The data set was measured from samples in Britain and Ireland, where a relatively limited area includes red sandstones from a range of stratigraphic ages, and the samples represent both first-cycle and multicycle erosion. Hence, they are a 


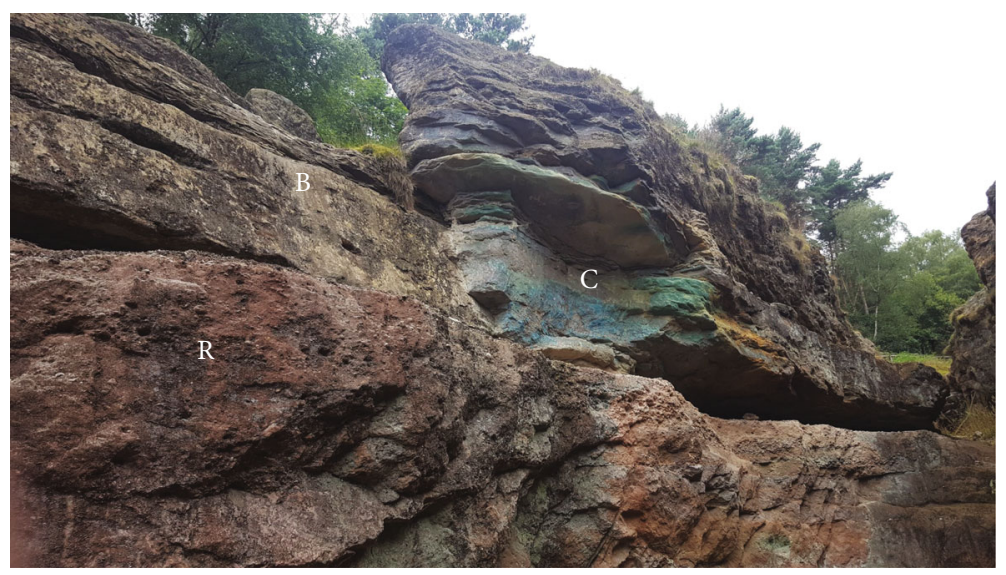

Figure 2: Sandstone exposure, Engine Vein, Alderley Edge copper mines. Bleached sandstone (B) overlies red sandstone (R) and passes into the copper-rich region $(\mathrm{C})$.

varied assemblage of sandstones suitable to best assess the chemistry of typical red grain coatings, rather than sandstones that are all associated with ore deposits. Samples were collected from 34 localities in Britain and Ireland (Figure 1), predominantly from red sandstones of Devonian, Permian, and Triassic age and deposited in fluvial or aeolian environments. Single samples of Neoproterozoic and Silurian red sandstone were collected. In addition, a sample of Carboniferous sandstone was collected, in which reddening had occurred at a relatively late stage, below the sub-Permian unconformity which caused widespread deep oxidation [27]. Red sandstone in dykes cutting down through the same unconformity [28] was collected at Berry Head, Torquay, England. A sample of Triassic sandstone at Maghera, Ireland, was chosen because it appeared to be enriched in iron by recent "iron pan" soil mineralization. Samples of red and bleached sandstone were collected for comparison from Alderley Edge, England (Figure 2), where bleaching is implicated in copper mineralization $[29,30]$. Three red sandstone samples from other parts of the world (China, Australia, USA) were analysed to test if the main data base is typical of other regions. Samples were chosen that could be disaggregated readily and were not cemented by minerals that sealed in the iron oxide and prevented dissolution. Disaggregated samples were sieved to isolate the sub-500 micron or sub250 micron fraction, depending on the predominant grain size. Sandstone from Gardenstown, Scotland, was separated into three size fractions, 500-250 micron, 250-125 micron, and sub-125 micron, to test if this factor might influence the compositions. Finally, a pure quartz sandstone with no iron oxide grain coatings (Cretaceous Lochaline Sandstone, Scotland) was analysed as a procedural blank.

Samples for acid leaching were added in weighed amounts of about $150 \mathrm{mg}$ to $4 \mathrm{~mL}$ aqua regia $(3: 1$ $\mathrm{HCl}: \mathrm{HNO}_{3}$, both analytical grade for trace elements) and boiled for 30 minutes at $95^{\circ} \mathrm{C}$ in a microwave. The liquid was then centrifuged, and the residue was subjected to a repeat of the procedure if any iron oxide remained undissolved (optical appearance). Only up to $20 \mathrm{wt}$.\% of each sample was dissolved by this procedure, leaving more than $80 \mathrm{wt}$ \% residue. The liquids from centrifuging were com- bined, and $\mathrm{V}, \mathrm{Co}, \mathrm{Cu}, \mathrm{Se}, \mathrm{U}, \mathrm{As}$, and $\mathrm{Pb}$ were measured by Triple Quad ICP-MS Agilent 8800 (Agilent Technologies), in the Trace Element Speciation Laboratory (TESLA), Department of Chemistry, at the Aberdeen University, UK. $10 \mu \mathrm{g} / \mathrm{kg} \mathrm{Rh}$ in $2 \% \mathrm{HNO}_{3}$ was used as an internal standard. The total leached element masses were determined (to an accuracy of $0.001 \mathrm{~g}$ ) and converted to concentrations of the original rock. Measurements were also taken from certified reference materials PACS-2 and BCSS-1 (marine sediments), and NIST2711a and NIST2709a (soils), and an absolute blank. Our data for V, Co, Cu, and Se for the NIST2711a and NIST2709a soils are consistent with the certificated data for the "acid-extractable" fraction. For the two sediments PACS-2 and BCSS-1, there is no certificated data for the "acid-extractable" fraction but only for the "whole rock" fraction, which also is in agreement with our data for $\mathrm{Co}, \mathrm{Cu}$, and Se, but show a higher value for V. Our acid leachable uranium, which had a recovery of $31-50 \%$ from certified totals, shows up to $70 \%$ totals from other ICPMS methods using PACS-2, and NIST SRM 2711a, 2709a [31, 32]. This indicates that the reported acid leachable uranium values most likely underestimate the content of uranium in the grain coatings.

Bulk analyses of Fe contents in all samples, and $\mathrm{V}$ and $\mathrm{Cu}$ in a subset of ten Permian and Triassic sandstone samples (available from another project that did not include the other samples), were made after multiacid digestion (perchloric, nitric, hydrofluoric, and hydrochloric acids) by ICP-MS.

Thin sections of a red sandstone from the Triassic of Northern Ireland (Lagan Valley borehole 4B, depth $30.7 \mathrm{~m}$ ) were mapped by laser ablation-ICP-MS. Elements were analysed using a LSX-213 G2+ laser ablation system (equipped with a Fast Washout 2-Volume HelEx II Cell and Aerosol Rapid Introduction System (ARIS), Teledyne CETAC Technologies) attached to a Triple Quad ICP-MS Agilent 8800 (Agilent Technologies), in TESLA at the Aberdeen University, UK. V (m/z 51, $0.01 \mathrm{~s}), \mathrm{Fe}(\mathrm{m} / \mathrm{z} 57,0.01 \mathrm{~s})$, Co (m/z 59, $0.01 \mathrm{~s}), \mathrm{Cu}(\mathrm{m} / \mathrm{z} 63,0.01 \mathrm{~s}), \mathrm{Se}(\mathrm{m} / \mathrm{z} \mathrm{78}, 0.1 \mathrm{~s})$, and $\mathrm{U}(\mathrm{m} / \mathrm{z}$ $238,0.1 \mathrm{~s}$ ) were measured (integration time). Analyses were performed in a helium atmosphere, at a repetition rate of $20 \mathrm{~Hz}$, a laser power of $6.02 \mathrm{~J} / \mathrm{cm}^{2}$, a spot diameter of $25 \mu \mathrm{m}$, and an ablation speed of $50 \mu \mathrm{m} / \mathrm{sec}$. Each line analysis 


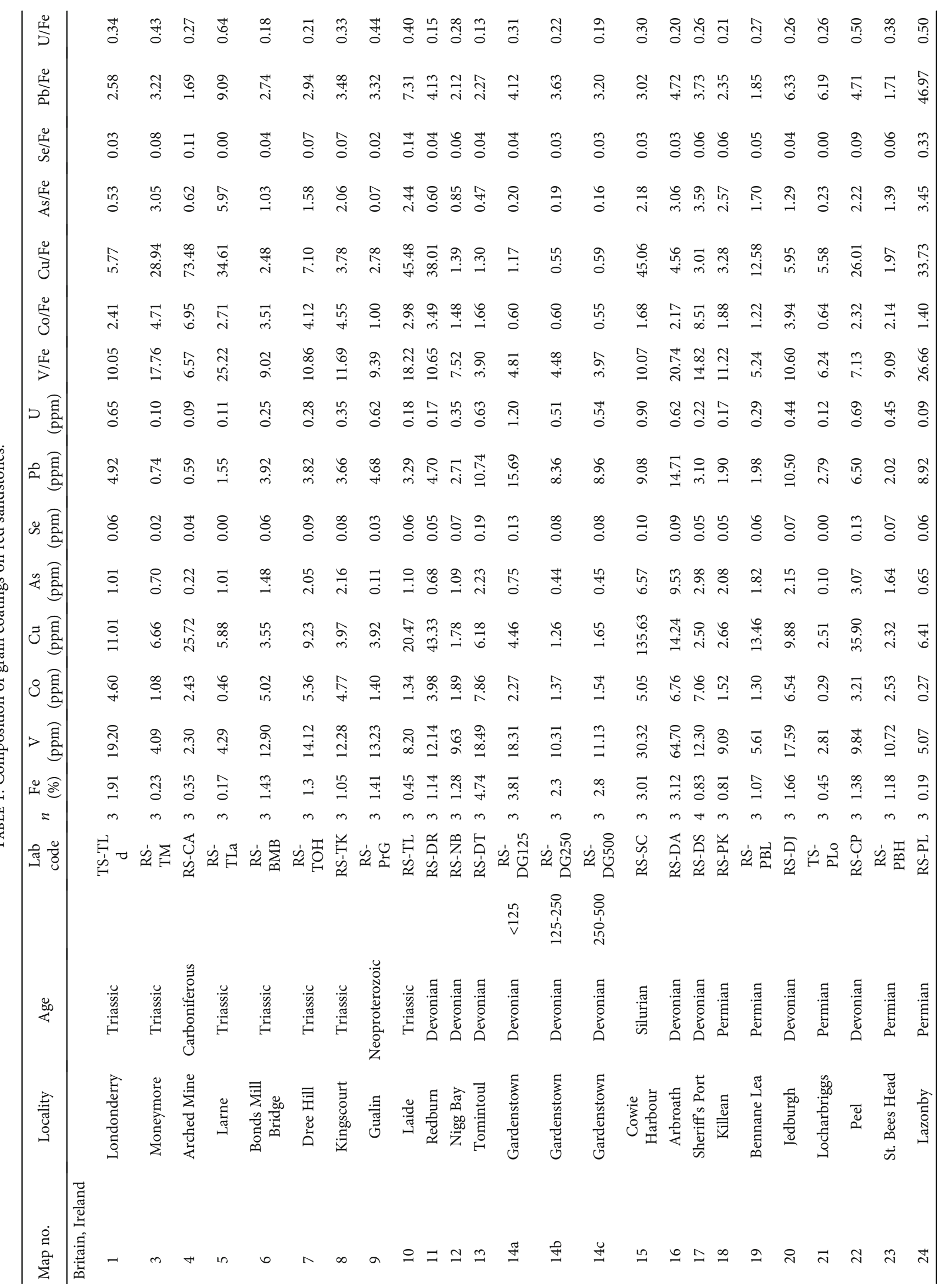




\begin{tabular}{|c|c|c|c|c|c|c|c|c|c|c|c|c|c|c|c|c|}
\hline$\sum_{5}^{\infty}$ & 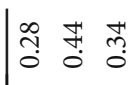 & ?! & के तิ & 苛 & $\overrightarrow{0}$ & $\stackrel{0}{0}$ & 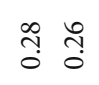 & ત્๋ તิ & $\tilde{3}$ & & $\stackrel{8}{0}$ & $\stackrel{\text { ஸ̊ }}{0}$ & $\stackrel{\overbrace{}}{0}$ & กิ & $\stackrel{\infty}{\stackrel{\infty}{-}}$ & $\tilde{\sigma}$ \\
\hline$\frac{0}{2}$ & 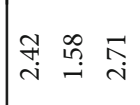 & $\stackrel{\infty}{\infty}$ & 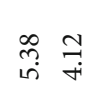 & iे & $\vec{m}$ & $\begin{array}{l}\text { in } \\
\text { aे }\end{array}$ & $\begin{array}{ll}+ & \infty \\
\infty & \infty \\
\dot{m} & m\end{array}$ & $\begin{array}{ll}\tilde{\sigma} & \overrightarrow{0} \\
\dot{m} & \infty\end{array}$ & $\stackrel{\text { qे }}{i}$ & & $\stackrel{\text { L }}{\stackrel{2}{+}}$ & $\stackrel{m}{+}$ & $\begin{array}{l}\overrightarrow{0} \\
\stackrel{\infty}{\hbar} \\
\stackrel{n}{n}\end{array}$ & $\begin{array}{l}\overrightarrow{\widehat{S}} \\
\text { ลे }\end{array}$ & 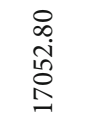 & $\begin{array}{l}8 \\
\infty \\
\infty \\
\infty \\
\infty \\
0\end{array}$ \\
\hline$\stackrel{\mathscr{L}}{\varpi}$ & {$\left[\begin{array}{lll}0 & \multicolumn{1}{c}{} \\
0 & 0 & 0 \\
0 & 0 & 0 \\
0\end{array}\right.$} & $\stackrel{0}{0}$ & $\stackrel{n}{n} \stackrel{4}{0}$ & $\stackrel{0}{0}$ & $\tilde{\Xi}$ & $\stackrel{0}{0}$ & $\stackrel{n}{0}:$ & $\begin{array}{l}\mathbb{3} \\
0\end{array}$ & $\stackrel{\leftrightarrow}{0}$ & & 8 & $\stackrel{0}{0}$ & $\stackrel{n}{\circ}$ & $\stackrel{n}{0}$ & $\stackrel{5}{0}$ & $\stackrel{\overbrace{}}{0}$ \\
\hline$\sum_{\frac{1}{4}}^{0}$ & 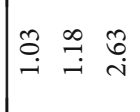 & $\stackrel{\text { i }}{\circ}$ & 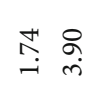 & $\stackrel{0}{\underset{I}{ت}}$ & $\stackrel{2}{m}$ & $\stackrel{\infty}{\text { in }}$ & $\stackrel{\text { Fे }}{F}$ & $\begin{array}{ll}\infty & 0 \\
0 & 0 \\
0 & \infty\end{array}$ & $\vec{\infty}$ & & 8 & $\stackrel{\infty}{\sharp}$ & $\stackrel{\vec{b}}{\wedge}$ & $\stackrel{\infty}{\infty}$ & $\begin{array}{l}\vec{b} \\
\stackrel{i}{ } \\
\vec{n} \\
\vec{N}\end{array}$ & $\begin{array}{l}\stackrel{0}{0} \\
\stackrel{\sim}{7} \\
\vec{\sim}\end{array}$ \\
\hline 芒 & 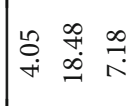 & $\underset{0}{\circ}$ & $\stackrel{\infty}{\infty} \stackrel{0}{\sigma}$ & 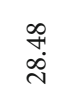 & 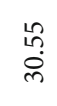 & $\begin{array}{l}\stackrel{\sim}{n} \\
\infty\end{array}$ & 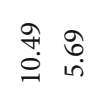 & 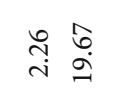 & $\begin{array}{l}\hat{B} \\
\stackrel{d}{i}\end{array}$ & & $\stackrel{8}{\circ}$ & $\underset{\sim}{F}$ & $\begin{array}{l}\infty \\
\infty \\
\stackrel{\infty}{n}\end{array}$ & $\underset{\stackrel{+}{+}}{\stackrel{q}{+}}$ & 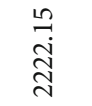 & $\begin{array}{l}\text { ô } \\
\text { ڤેं } \\
\text { సे }\end{array}$ \\
\hline$\frac{\pi}{3}$ & 㹊 & $\stackrel{?}{m}$ & $\stackrel{\overbrace{}}{\stackrel{P}{i}} \stackrel{\infty}{\stackrel{\infty}{n}}$ & $\stackrel{\infty}{\infty}$ & $\frac{6}{6}$ & $\stackrel{\infty}{\infty}$ & ते $\stackrel{m}{i}$ & సે $\overrightarrow{0}$ & $\stackrel{\infty}{\stackrel{\infty}{+}}$ & & $\underset{⿱}{\stackrel{\sharp}{N}}$ & 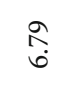 & 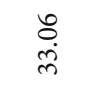 & 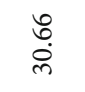 & $\begin{array}{l}\infty \\
\stackrel{+}{+} \\
\stackrel{+}{+}\end{array}$ & ڤू \\
\hline$\stackrel{\pi}{>}_{>}^{0}$ & 点 & $\stackrel{\vec{b}}{\circ}$ & 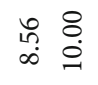 & $\begin{array}{l}\stackrel{0}{0} \\
\stackrel{\infty}{\rightarrow}\end{array}$ & f & ले & $\stackrel{\circ}{\circ}=$ & $\ln _{\infty}^{\infty} \stackrel{N}{N}$ & 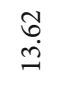 & & $\begin{array}{l}\infty \\
\infty \\
\stackrel{0}{-}\end{array}$ & ò & 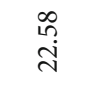 & 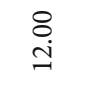 & $\stackrel{\circ}{\circ}$ & 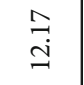 \\
\hline ○ 彥 & 공 & 今ે & $\stackrel{10}{0}$ & $\dddot{0}$ & సิ & 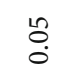 & $\stackrel{\infty}{\infty} \stackrel{\infty}{0}_{0}^{\infty}$ & $\stackrel{0}{0} \stackrel{10}{0}$ & $\stackrel{5}{0}$ & & $\ddot{\circ}$ & $\stackrel{\infty}{\infty}$ & $\stackrel{\infty}{\stackrel{\infty}{0}}$ & $\stackrel{H}{0}$ & ñ & กิ \\
\hline ฉ & 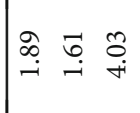 & in & 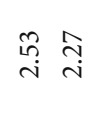 & 予 & $\overbrace{b}^{n}$ & 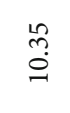 & 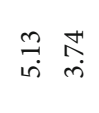 & $\stackrel{f}{\rightarrow} \stackrel{0}{+}$ & ָุ & & $\stackrel{\sharp}{0}$ & $\stackrel{\widehat{m}}{\stackrel{+}{+}}$ & $\begin{array}{l}m \\
\stackrel{2}{\infty} \\
\infty\end{array}$ & $\begin{array}{l}\text { H } \\
\text { in } \\
\text { ñ }\end{array}$ & 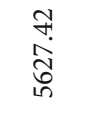 & 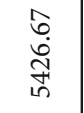 \\
\hline \& & 若 & $\dddot{0}$ & $\stackrel{0}{0} 0$ & $\stackrel{\text { L }}{0}$ & $\stackrel{0}{0}$ & $\stackrel{8}{0}$ & $\stackrel{0}{0}:$ & $\stackrel{0}{0} \stackrel{0}{0}$ & $\ddot{0}$ & & $\stackrel{8}{0}$ & $\vec{\Im}$ & $\stackrel{\circ}{\circ}$ & $\stackrel{\infty}{\circ}$ & $\stackrel{0}{0}$ & $\hat{\circ}$ \\
\hline स & 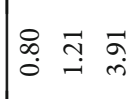 & i. & 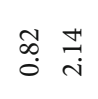 & $\stackrel{\hat{n}}{\hat{m}}$ & $\stackrel{+}{\stackrel{5}{n}}$ & $\stackrel{\infty}{-\infty}$ & 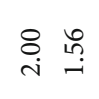 & $\vec{m} \hat{0}$ & $\stackrel{\circ}{\stackrel{0}{i}}$ & & $\stackrel{0}{0}$ & $\stackrel{\bullet}{\circ}$ & $\stackrel{H}{\sharp}$ & $\stackrel{\infty}{\underset{+}{+}}$ & $\begin{array}{l}\text { in } \\
\text { on } \\
\text { م }\end{array}$ & $\begin{array}{l}0 \\
\infty \\
\infty \\
0 \\
0\end{array}$ \\
\hline 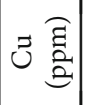 & 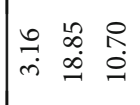 & 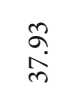 & $\stackrel{\circ}{\circ}$ & $\begin{array}{l}\infty \\
\infty \\
\infty \\
\infty\end{array}$ & 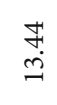 & $\underset{\substack{i \\
i}}{ }$ & ठे & $\begin{array}{ll}\stackrel{+}{0} & \overrightarrow{0} \\
\dot{0} & \stackrel{\Xi}{=}\end{array}$ & $\stackrel{m}{m}$ & & ? & 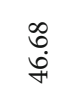 & $\begin{array}{l}\hat{a} \\
\hat{\vdots}\end{array}$ & 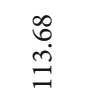 & $\vec{m}$ & $\begin{array}{l}\vec{n} \\
\infty \\
\infty \\
\infty\end{array}$ \\
\hline ن & 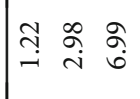 & $\vec{b}$ & $\stackrel{f}{\stackrel{f}{0}}$ & $\begin{array}{l}\infty \\
\infty \\
\infty \\
0\end{array}$ & $\vec{i}$ & $\stackrel{0}{0}$ & 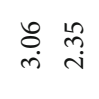 & $\exists \stackrel{n}{0}$ & $\stackrel{\widetilde{\pi}}{i}$ & & $\stackrel{0}{\circ}$ & 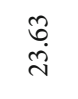 & $\begin{array}{l}8 \\
\dot{7}\end{array}$ & $\stackrel{\stackrel{n}{q}}{\underline{n}}$ & $\underset{\vec{q}}{\stackrel{\vec{q}}{ }}$ & 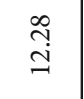 \\
\hline 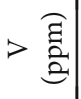 & 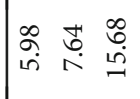 & $\begin{array}{l}\hat{L} \\
\infty \\
\infty \\
\infty\end{array}$ & $\begin{array}{ll}\text { do } \\
\text { o }\end{array}$ & 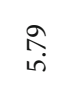 & $\overrightarrow{\vec{f}}$ & $\stackrel{\sim}{\sim}$ & $\begin{array}{ll}\tilde{O} & \hat{0} \\
\text { İ } & 0\end{array}$ & 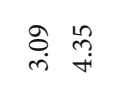 & $\stackrel{\infty}{\circ}$ & & $\stackrel{\text { ঙ̊ }}{0}$ & $\underset{\stackrel{\infty}{+}}{\stackrel{+}{0}}$ & $\begin{array}{l}8 \\
\infty \\
i \\
i\end{array}$ & $\underset{\wp}{0}$ & $\stackrel{m}{m}$ & $\ddot{n}$ \\
\hline 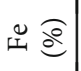 & 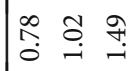 & $\stackrel{n}{\sim}$ & fy & $\vec{m}$ & $\underset{H}{H}$ & $\stackrel{\substack{m \\
0}}{o}$ & $\stackrel{\leftrightarrow}{ت}$ & ĉn & กี่ & & $\ddot{0}$ & $\underset{\substack{\infty \\
m}}{ }$ & $\stackrel{\stackrel{H}{I}}{\sim}$ & $\stackrel{n}{n}$ & $\stackrel{m}{m}$ & ? \\
\hline$=$ & $m \sim n$ & n & $n N$ & n & $n$ & n & & $n n$ & $n$ & & $n$ & $m$ & n & m & n & $m$ \\
\hline 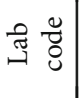 & 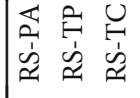 & 宅 & 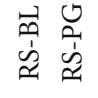 & ن & 官 & 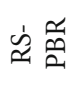 & & 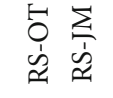 & $\approx$ & & ๓े & & & 宅密 & $\dot{\theta}$ & 宅皁 \\
\hline & & & & & & & & & & & & & $\ddot{\mathscr{\Xi}}$ & $\widetilde{\mathscr{U}}$ & 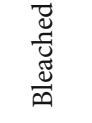 & 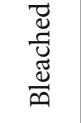 \\
\hline$\stackrel{\circ}{<}$ & 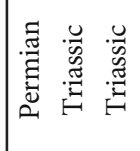 & 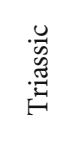 & 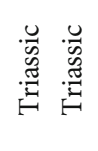 & 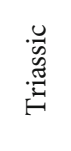 & 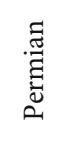 & 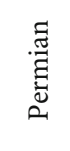 & & 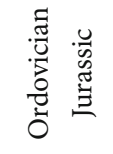 & 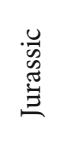 & & 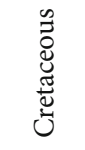 & 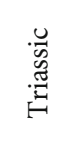 & 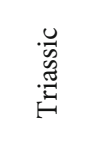 & 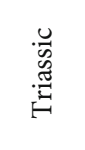 & 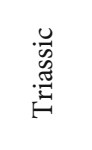 & 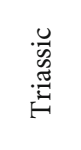 \\
\hline 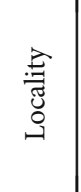 & 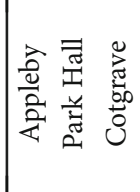 & 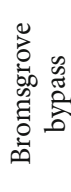 & 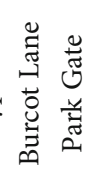 & $\begin{array}{l}\tilde{y} \\
\frac{\tilde{u}}{0} \\
0 \\
0\end{array}$ & 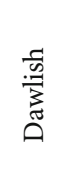 & 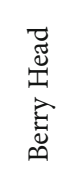 & & 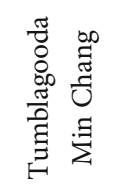 & 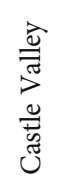 & & 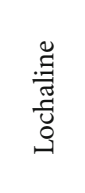 & 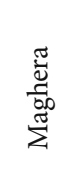 & 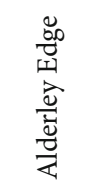 & 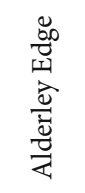 & 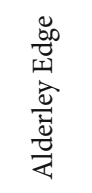 & 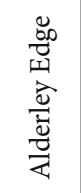 \\
\hline 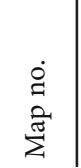 & $\stackrel{\sim}{\sim} \curvearrowright \infty$ & নे & $\ddot{m} \vec{m}$ & లె & $m$ & $\ddot{m}$ & 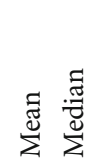 & $\xi \xi$ & & 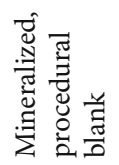 & 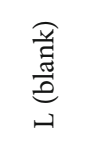 & $\sim$ & $\stackrel{\widetilde{N}}{\sim}$ & iి & ֻั & $\vec{d}$ \\
\hline
\end{tabular}



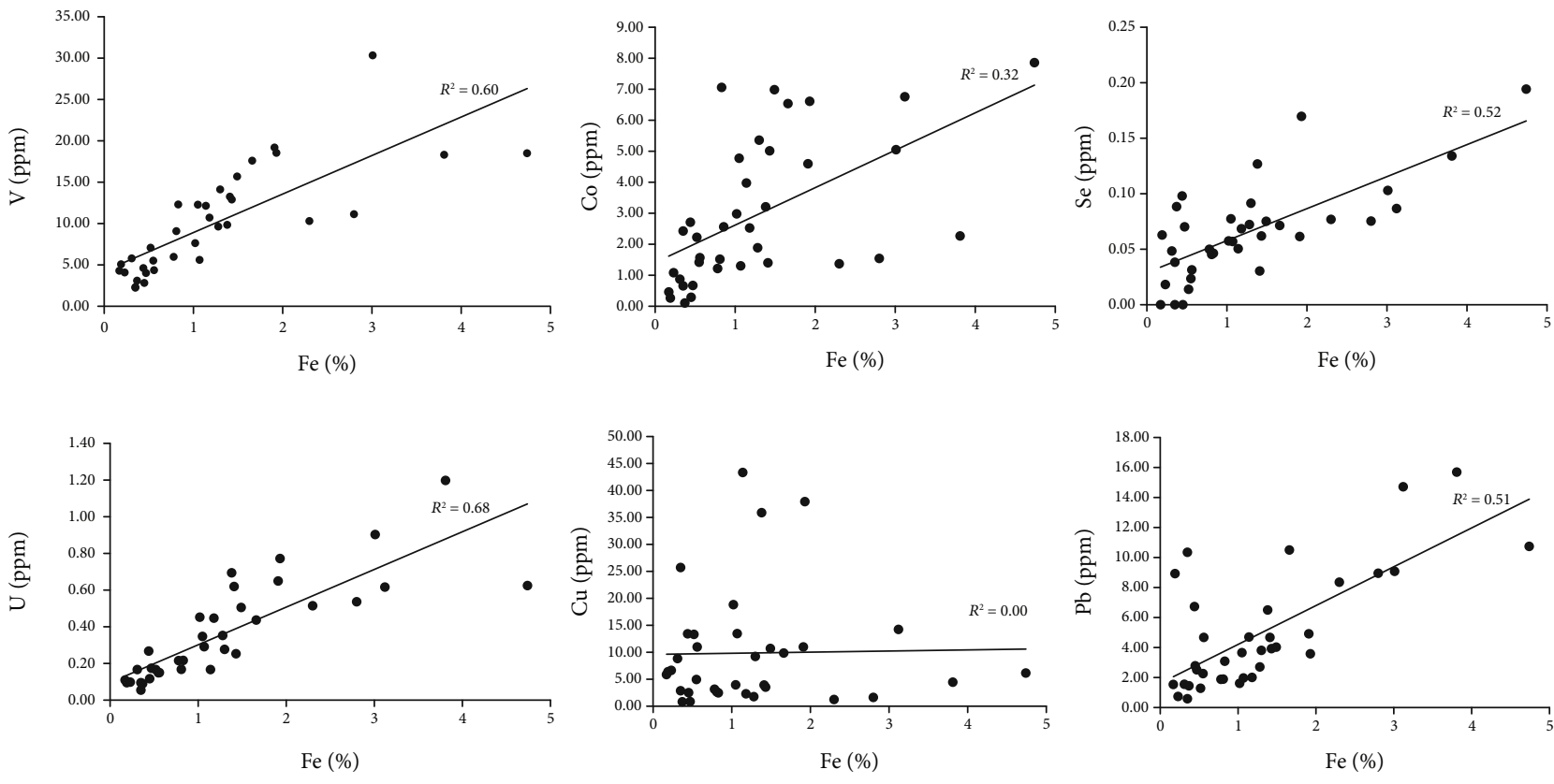

FigURE 3: Cross-plots for whole rock Fe content against content of trace elements measured by acid extraction. Single samples omitted from cross-plots against V (locality 16) and $\mathrm{Cu}$ (locality 15) where they are distinct outliers. Five of six plots show positive correlation, while plot against $\mathrm{Cu}$ shows no correlation.

included $11 \mathrm{~s}$ background analysis followed by $20-30$ s laser ablation time. Hydrogen was used in the collision reaction cell of ICP-MS/MS with a flow of $3.5 \mathrm{~L} / \mathrm{min}$ to remove polyatomic interferences. Argon was used as dilution and carrier gas in ICP-MS/MS. The instrument was optimized for minimal molecular oxide species (i.e., ${ }^{232} \mathrm{Th}^{16} \mathrm{O} /{ }^{232} \mathrm{Th}<0.3 \%$ ). Optimization of the instrument was performed by ablating the glass reference NIST612. Two reference materials of MRM MASS- 1 from USGS and UQAC-FeS- 1 from SLIM were used as calibration standards only.

\section{Results}

Each of the red sandstone samples processed by acid removal of grain coatings was leached, indicating liberation of iron and associated trace elements to solution. Analysis of the solutions, converted to concentrations of whole rock, confirmed that trace elements had been liberated (Table 1). The absolute blank and procedural blank yielded negligible contents (Table 1). Data for certified reference materials and all standard deviations are given in the Supplementary Information (Table S1). SEM observations confirmed that while iron oxides were removed by acid leaching, the substrate grains remained unaffected. Ranges of elements determined included 0.8 to $135.6 \mathrm{ppm} \mathrm{Cu}$ (mean $14.0 \mathrm{ppm}$, median $6.3 \mathrm{ppm}, \quad n=37$ ), 2.2 to $64.7 \mathrm{ppm} \mathrm{V}$ (mean $12.0 \mathrm{ppm}$, median $10.1 \mathrm{ppm}, n=37), 0.05$ to $1.2 \mathrm{ppm} \mathrm{U}$ (mean $0.4 \mathrm{ppm}$, median $0.3 \mathrm{ppm}, n=37$ ), and 0.3 to $7.9 \mathrm{ppm}$ Co (mean $3.1 \mathrm{ppm}$, median $2.4 \mathrm{ppm}, n=37$ ), excluding the copper-mineralized locality at Alderley Edge and the locality at Maghera influenced by soil mineralization. The small set of samples from other countries gave values comparable with those from Britain and Ireland.

The bleached sandstone from Alderley Edge yielded mean acid leach values higher than the red sandstones there. All the values for $\mathrm{Cu}, \mathrm{Co}$, and $\mathrm{Pb}$ at Alderley Edge are much higher than in other samples. The sandstone from Maghera enriched by soil mineralization contains relatively high levels of $\mathrm{V}, \mathrm{Co}, \mathrm{Cu}, \mathrm{Pb}$, and $\mathrm{U}$. Comparison of the grain size fractions from Gardenstown showed that the composition of the two coarser fractions that were purely sand size was similar, but the finer size fraction that included mud had higher contents of trace elements.

The subset of Permian and Triassic samples measured for both whole rock composition and grain coating leach composition have mean compositions of $11 \mathrm{ppm} \mathrm{V}$ and $7.6 \mathrm{ppm} \mathrm{V}$ and $6.1 \mathrm{ppm} \mathrm{Cu}$ and $5.2 \mathrm{ppm} \mathrm{Cu}$, respectively.

Whole rock Fe contents range up to $4.74 \%$. The contents of $\mathrm{V}, \mathrm{Co}, \mathrm{Se}, \mathrm{U}$, and $\mathrm{Pb}$ show moderate positive correlation with $\mathrm{Fe}$ content ( $R^{2}$ values 0.32 to 0.69 ; Figure 3$)$. There is no correlation between $\mathrm{Fe}$ and $\mathrm{Cu}$. Distributions of values within the data set show a bias towards lower values, but in the case of $\mathrm{Cu}$, there is an additional group of higher values (Figure 4).

The LA-ICP-MS maps of a quartz grain with surrounding iron oxide coating in the thin section of red sandstone show a clear contrast between sand grain and grain coating (Figure 5). The grains exhibit negligible (less than $1 \mathrm{ppm}$ ) concentrations of $\mathrm{V}$ and $\mathrm{Co}$, while the surrounding iron oxide shows $\mathrm{V}$ and $\mathrm{Co}$ at up to $120 \mathrm{ppm}$ and $60 \mathrm{ppm}$, respectively. 


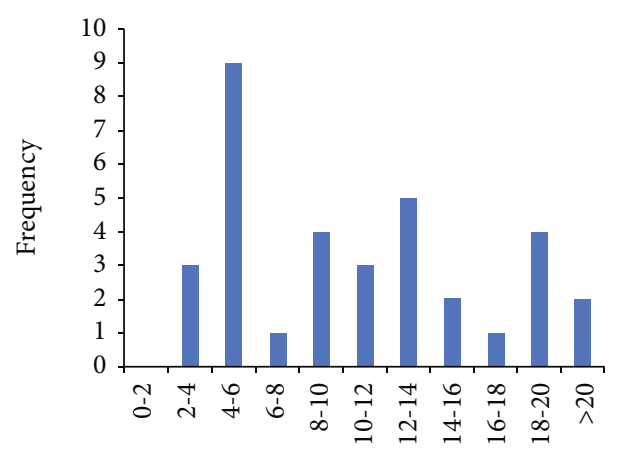

$\mathrm{V}(\mathrm{ppm})$

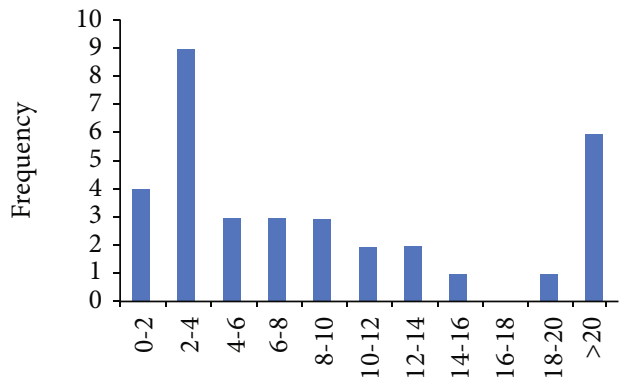

$\mathrm{Cu}(\mathrm{ppm})$

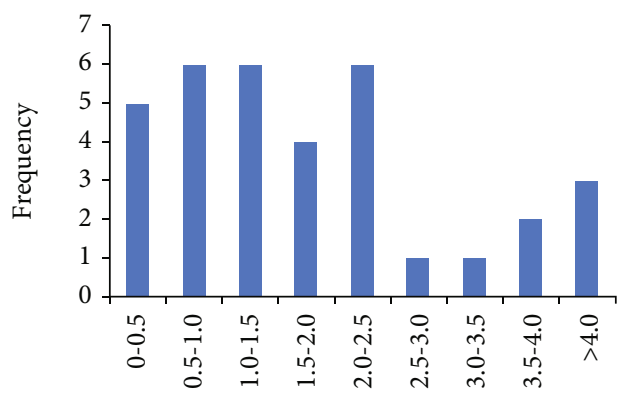

As (ppm)

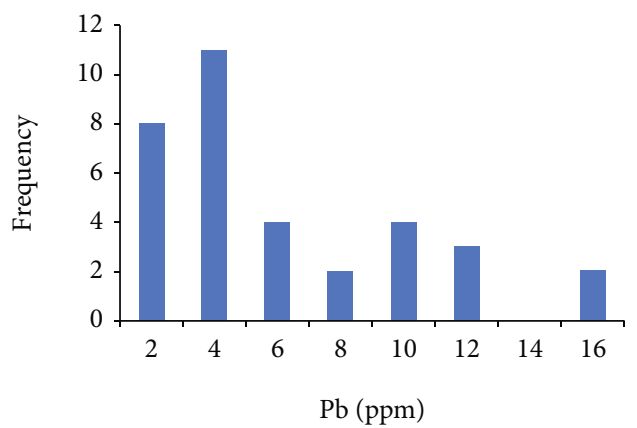

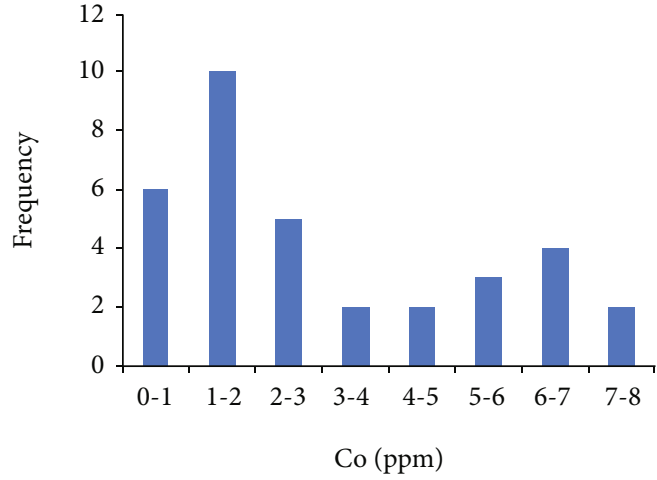

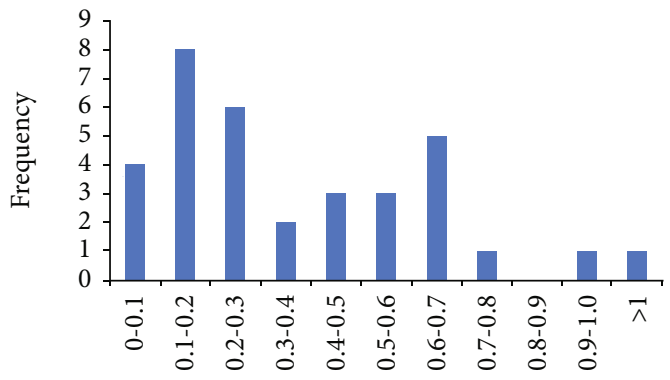

$\mathrm{U}(\mathrm{ppm})$
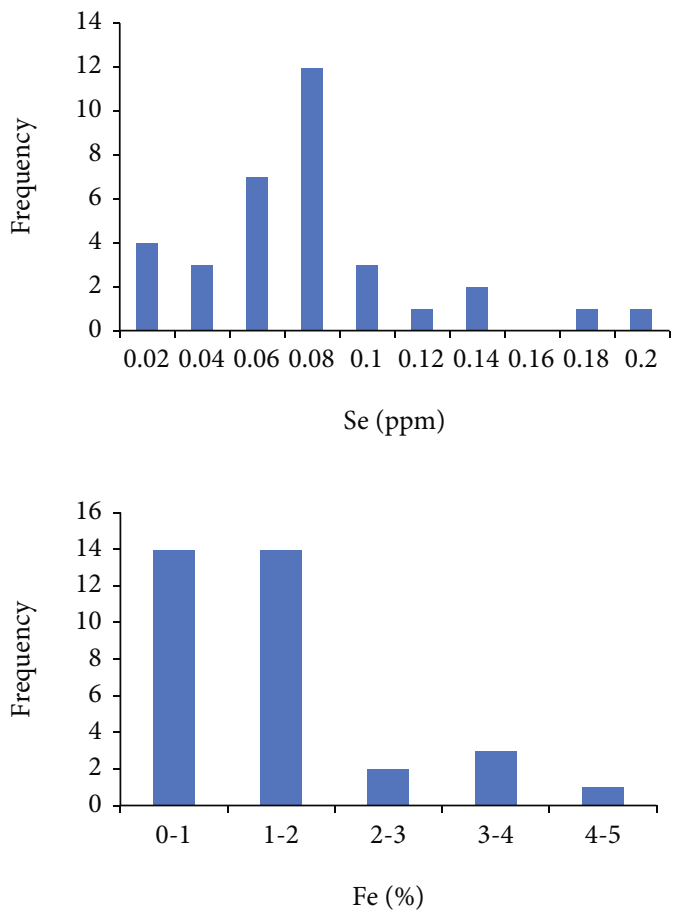

FIGURE 4: Distribution of values for trace elements and Fe within data set.

\section{Discussion}

4.1. Compositions of Grain Coatings. Previous proposals of a model of mineralization from leached grain coatings are based on the circumstantial occurrence of bleached sandstones in ore fields $[14,17,18]$, distal reprecipitation of leached metals $[6,7]$, or whole rock data [11]. Measurements of some trace elements in iron oxide grain coatings were made by [33], including $\mathrm{U}$ and $\mathrm{V}$ and rare earth elements but not $\mathrm{Cu}, \mathrm{Co}$, or Se. The data set reported here for grain coatings is more comprehensive than hitherto. Variations in compositions of grain coatings are assessed here in terms of the iron content and the stratigraphic age.

The relatively high values of several metals within the soil-influenced sample from Maghera indicate that enrichment in iron oxides can occur on a geologically short time 

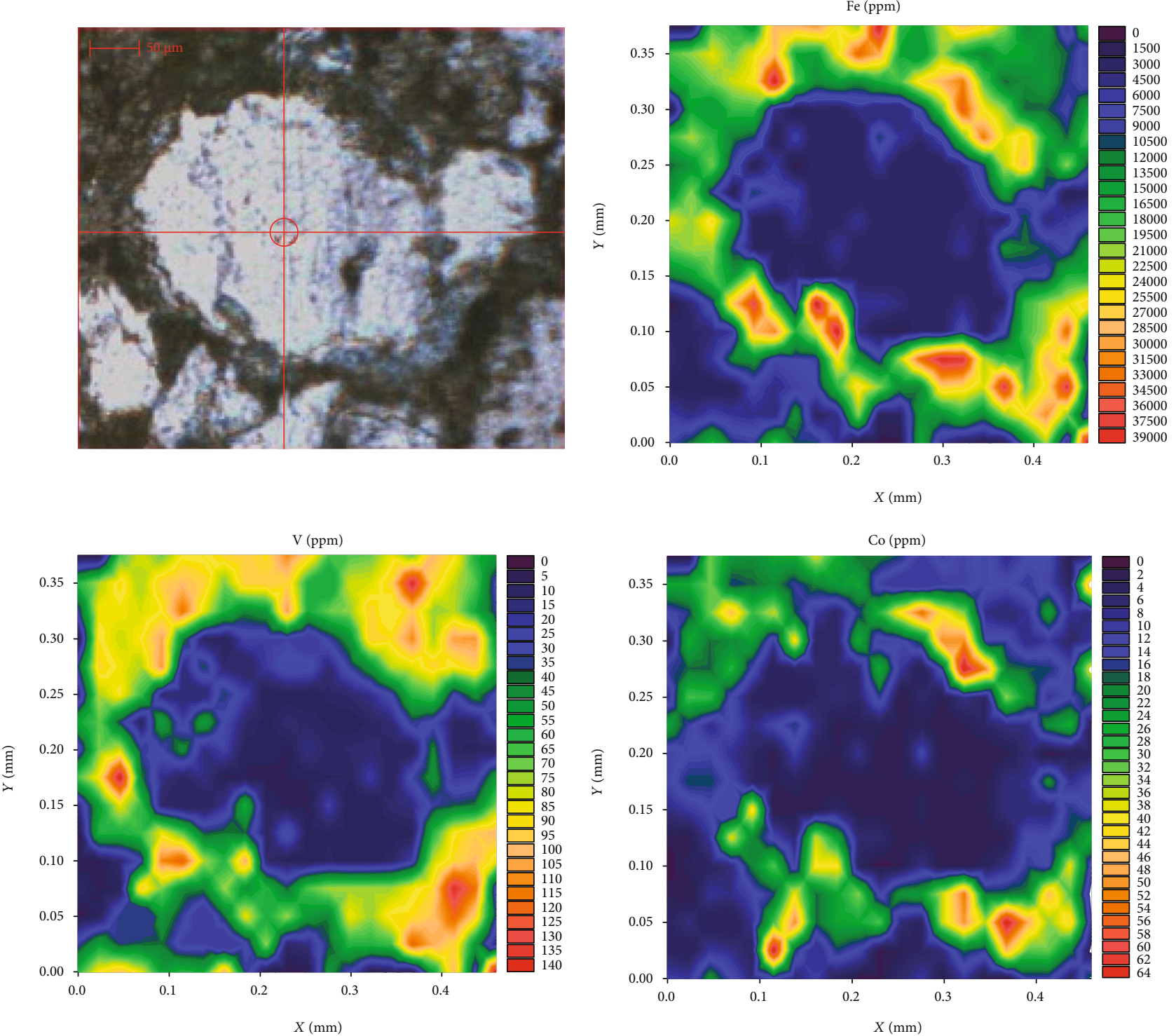

Figure 5: LA-ICP-MS maps for Fe, V, and Co, Triassic sandstone, Lagan Valley borehole 4B, oxide grain coating, marked by very high Fe levels, shows up to $120 \mathrm{ppm} \mathrm{V}$ and $60 \mathrm{ppm} \mathrm{Co}$, but negligible contents in the grain.

scale. Similar enrichment at the surface is the basis of mineral exploration using metal anomalies in iron oxide precipitates in the surface environment $[34,35]$. The early incorporation of trace elements is consistent with the model for metal release by mineral alteration during early diagenesis $[2,9]$.

Most sandstones have $\mathrm{Fe}$ contents in the range of 0.17 to $4.74 \%$ (mean $1.27 \%$ ). The mean concentrations of aqua regia leachable elements from the unmineralized red sandstones are $14.0 \mathrm{ppm} \mathrm{Cu}, 12.0 \mathrm{ppm} \mathrm{V}, 0.4 \mathrm{ppm} \mathrm{U}$, and $3.1 \mathrm{ppm}$ Co. The median values are comparable, except in the case of copper which has a much lower median value of $6.3 \mathrm{ppm}$. In contrast, the pure quartz sandstone contains negligible contents of iron or trace elements (less than $1 \%$ of the mean values). The broad correlation of iron and other metals in the whole rock data (Figure 3) suggests that the residence for much of the trace metal is within the iron oxide grain coatings. This is consistent with the LA-ICP-MS maps, which show trace elements only in the grain coatings and not in the substrate grains. The lack of correlation between $\mathrm{Cu}$ and $\mathrm{Fe}$ (Figure 3) may reflect the incorporation of $\mathrm{Cu}$ into other phases such as aluminosilicates, as observed elsewhere in red sandstones $[17,36]$.

The measurements made are of metal that is readily leachable from the sandstone, rather than the total metal content of the sandstones. Some metal within detrital grains may not be readily accessible to the leaching fluids, whereas the metal in iron oxide grain coatings is amenable to leaching. However, a comparison of whole rock data and grain coating leach data for a subset of ten red sandstone samples analysed for $\mathrm{V}$ and $\mathrm{Cu}$ showed that over $70 \%$ and $85 \%$, respectively, of the trace elements reside in the grain coatings and is leachable. Notably, the mean values of metals in the grain coatings are not anomalously high compared to typical rock compositions, but the occurrence of the metals in abundant thin coatings makes them relatively available. 

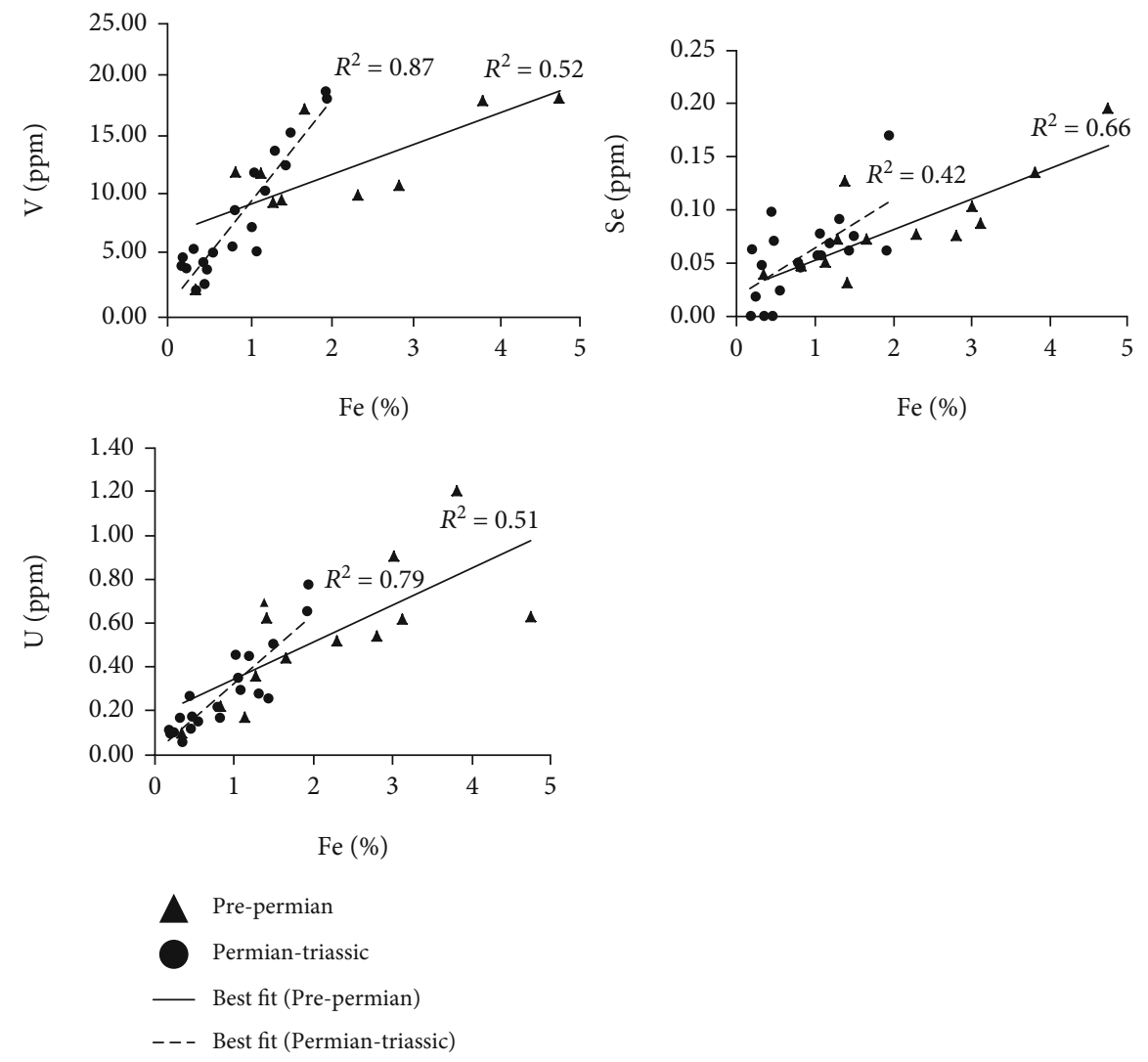

Figure 6: Comparison of acid leach compositions for red sandstones of pre-Permian age and Permian-Triassic age. Higher V values occur in pre-Permian samples. Data for other elements do not show a relationship with stratigraphy. Mineralized samples were omitted.

The aqua regia used to remove the iron oxide grain coatings is a more acidic and oxidizing solvent than would occur in nature, and the dissolution temperature $\left(95^{\circ} \mathrm{C}\right)$ in the laboratory is higher, so shows the total potential that can be released, rather than what might be released during a single fluid flow event. Nevertheless, given that ore deposits may form over a period of millions of years (Brown, 2009), the total potential is an appropriate value to measure. Red bed sandstones commonly exhibit zones that are completely bleached, indicating that the iron oxide grain coating is completely removed over time.

Comparison of metal values with stratigraphic age shows that values of $\mathrm{V}$ and $\mathrm{U}$ are higher in the red sandstones of pre-Permian age than in those of Permian and Triassic age (Figures 6 and 7). The mean values for $\mathrm{V}$ are $17.7 \mathrm{ppm}$ $(n=13)$ and $6.7(n=21)$, respectively. The mean values for $\mathrm{U}$ are 0.54 and 0.28 , respectively. In contrast, values for the other elements show no relationship with stratigraphic age. The range of pre-Permian values for $\mathrm{Cu}$ is greater than for Permo-Triassic values, but the mean values are similar (Figure 7). The pre-Permian samples are mostly from northern Britain, where the provenance is in the Caledonide basement and sandstones represent first cycle erosion. They are therefore more likely to contain mineralogically immature grains than the Permo-Triassic sandstones which are commonly derived from multiple cycles of erosion, especially the aeolian Permian sandstones. The immature grains would have yielded trace metals during diagenetic alteration, whereas quartz-rich sandstones have much less potential. However, the higher contents in pre-Permian sandstones also reflect higher Fe contents (Figure 6). In fact, the $\mathrm{V} / \mathrm{Fe}$, $\mathrm{Se} / \mathrm{Fe}$, and $\mathrm{U} / \mathrm{Fe}$ ratios are lower in the pre-Permian samples. The lower ratios in older sandstones could indicate that some of the trace elements have been released from the grain coatings, at a higher thermal maturity than in the younger sandstones. With thermal maturation, the crystallinity of haematite increases [37], and the capacity to adsorb trace metals would decrease with recrystallization [38], although changes in surface area during alteration could complicate the effect on adsorption. The contrast with age may be more evident in the case of $\mathrm{V}, \mathrm{Se}$, and $\mathrm{U}$ because of very ready redox-controlled mobility $[39,40]$, which is also evident in the predominance of these elements in diagenetic reduction spheroids $[41,42]$. On the other hand, vanadian haematite containing percent level $\mathrm{V}$ is identified in some ore deposits, so this can occur as a stable phase $[43,44]$.

4.2. Ore Fluids. These measurements allow us to calculate the volume of rock required to supply metal to a large ore deposit (Table 2). For a sandstone of density $2.5 .10^{3} \mathrm{kgm}^{-3}$ (values typically range from 2.2 to $2.8 .10^{3} \mathrm{kgm}^{-3}$ ), a metal availability of $10 \mathrm{ppm}$ equates to $1 \mathrm{~kg}$ of metal from $40 \mathrm{~m}^{3}$ ( $1 \mathrm{Mt}$ from $\left.40 \mathrm{~km}^{3}\right)$ or $2.5 \mathrm{~kg}$ of metal from $100 \mathrm{~m}^{3}\left(2.5 .10^{4}\right.$ tonnes from $1 \mathrm{~km}^{3}$ ) rock. Aquifer volumes for Triassic red beds, measured for potential $\mathrm{CO}_{2}$ storage, range from hundreds to thousands of $\mathrm{km}^{3}$ [45-47]. For $10 \mathrm{ppm}$ metal availability, an aquifer of 


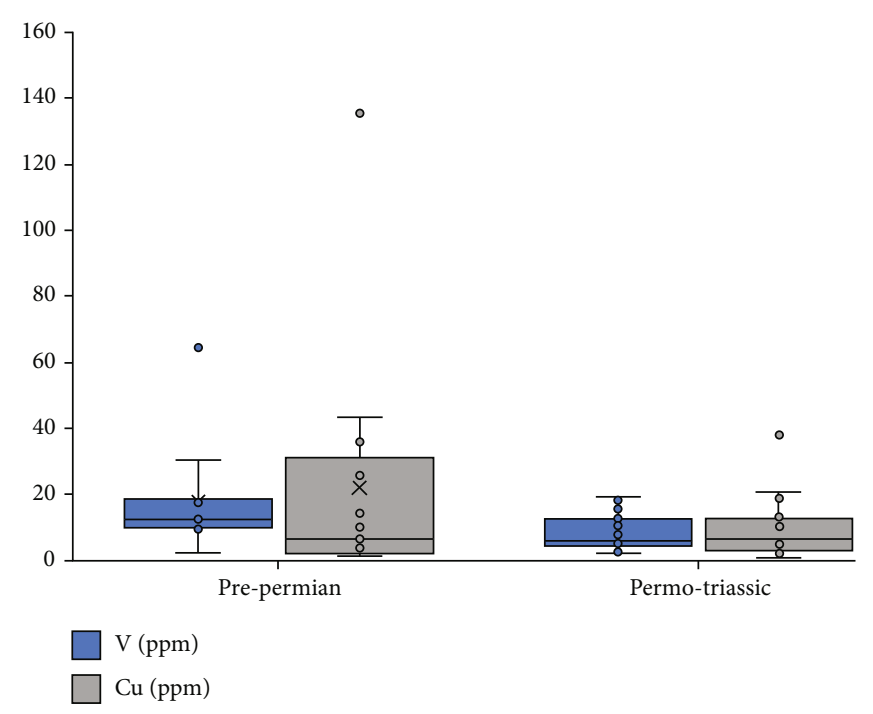

(a)

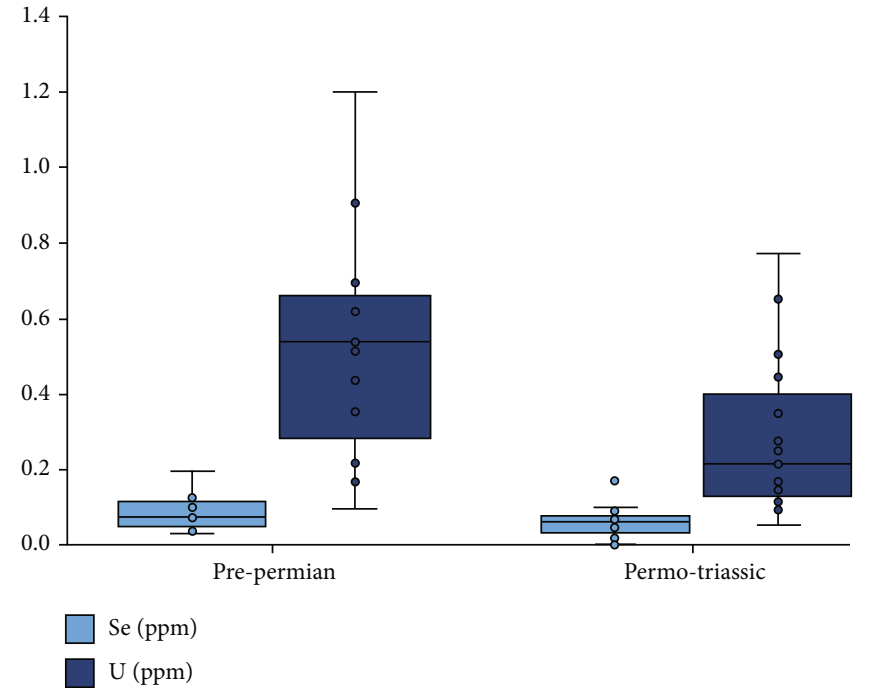

(b)

Figure 7: Box-and-whisker plots for V, Cu, Se, and U values in pre-Permian and Permo-Triassic values. Data show higher Permian samples.

TABLE 2: Metal availability from grain coatings relative to requirements for ore deposits.

\begin{tabular}{lccccc}
\hline Element & $\begin{array}{c}\text { Median } \\
\text { available (ppm) }\end{array}$ & $\begin{array}{c}\text { Size most sandstone- } \\
\text { hosted ore deposits }\end{array}$ & $\begin{array}{c}\text { Available metal } \\
\text { in } 10^{3} \mathrm{~km}^{3}\end{array}$ & $\begin{array}{c}\text { \% of available metal in } 10^{3} \mathrm{~km}^{3} \\
\text { needed for ore deposits }\end{array}$ & $\begin{array}{c}\text { Aquifer volume needed } \\
\text { for ore deposits }\end{array}$ \\
\hline $\mathrm{Cu}$ & 6.29 & Mean $22 \mathrm{Mt}$; many $1 \mathrm{Mt}$ & $15.73 \mathrm{Mt}$ & $\begin{array}{c}140 \% \text { for } 22 \mathrm{Mt} \\
6 \% \text { for } 1 \mathrm{Mt}\end{array}$ & $\begin{array}{c}1399 \mathrm{~km}^{3} \text { for } 22 \mathrm{Mt} \\
64 \mathrm{~km}^{3} \text { for } 1 \mathrm{Mt}\end{array}$ \\
$\mathrm{U}$ & 0.28 & 0.1 to $10 \mathrm{kt}$ & $0.7 \mathrm{Mt}$ & 0.01 to $1.4 \%$ & 0.1 to $14 \mathrm{~km}^{3}$ \\
$\mathrm{~V}$ & 10.07 & 1 to $100 \mathrm{kt}$ & $25.2 \mathrm{Mt}$ & 0.004 to $0.4 \%$ & 0.04 to $4 \mathrm{~km}^{3}$ \\
$\mathrm{Co}$ & 2.35 & 5 to $500 \mathrm{kt}$ (all ores) & $5.88 \mathrm{Mt}$ & 0.09 to $8.5 \%$ & 0.9 to $85 \mathrm{~km}^{3}$ \\
\hline
\end{tabular}

$10^{3} \mathrm{~km}^{3}$ would yield $25 \mathrm{Mt}$ metal. The measured median 6.3 ppm $\mathrm{Cu}$ available would yield $15.7 \mathrm{Mt}$ in $10^{3} \mathrm{~km}^{3}$. To put these values in context, the $\mathrm{Cu}$ resources in the worldclass Chu-Sarysa Basin, Kazakhstan, consist of a huge $22 \mathrm{Mt}$ deposit and smaller deposits of about $1 \mathrm{Mt}$ [14]. The mean size of sediment-hosted $\mathrm{Cu}$ deposits is also $22 \mathrm{Mt}$ [48], equivalent to $140 \%$ of the $\mathrm{Cu}$ available in $10^{3} \mathrm{~km}^{3}$. Previous calculations for the Triassic red beds of central England concluded that 1 ppm Cu from $340 \mathrm{~km}^{3}$ could yield over $0.8 \mathrm{Mt} \mathrm{Cu}$ [49], and $2 \mathrm{ppm} \mathrm{Cu}$ from $3300 \mathrm{~km}^{3}$ Triassic sandstone could yield $15 \mathrm{Mt} \mathrm{Cu}$ [50].

Most sandstone-hosted $\mathrm{U}$ deposits contain 0.1 to $10 \mathrm{kt} \mathrm{U}$ [51]. This represents 0.01 to $1.4 \%$ of the $U$ available from $10^{3} \mathrm{~km}^{3}$ (Table 2). Most sandstone-hosted V deposits contain 1 to $100 \mathrm{kt} \mathrm{V}$ [52], which would require 0.004 to $0.4 \%$ of the $\mathrm{V}$ available from $10^{3} \mathrm{~km}^{3}$ (Table 2). Most types of Co deposit contain 5 to $500 \mathrm{kt}$ Co [53], requiring 0.09 to $8.5 \%$ of the Co that could be released in $10^{3} \mathrm{~km}^{3}$.

The data for red and bleached sandstones at Alderley Edge, where $\mathrm{Cu}$ mineralization is attributed to the dissolution of grain coatings by reducing brines including hydrocarbons $[30,50]$, show variable patterns for different elements. The bleached sandstones have lower values of $\mathrm{V}$ and $\mathrm{Co}$, but much higher values of $\mathrm{Cu}$, As, and $\mathrm{Pb}$. However, the high $\mathrm{Cu}$ values in the bleached sandstones indicate that the $\mathrm{Cu}$ was not exported from the system and has another residence.
The bleached sandstones have a lower iron content, but they contain a range of secondary $\mathrm{Cu}$ minerals [29]. The lower values of $\mathrm{V}$, at least, might reflect uptake by migrating hydrocarbons.

Multiple factors will control metal release, including $\mathrm{pH}$, Eh, temperature, and pore fluid chemistry [10]. The model considered here envisages the dissolution of grain coatings by acidic fluids, particularly migrating oil and gas. Largescale hydrocarbon migration, evidenced by widespread oil residues, therefore, contributes to the exploration for sandstone-hosted ore deposits $[16,17]$. Most oil is generated $<130^{\circ} \mathrm{C}$, and it is likely that related removal of iron oxide grain coatings occurs at shallower depths and lower temperatures. This implies the removal of coatings and liberation of trace metals at depths of less than $4 \mathrm{~km}$. At these depths, there is still adequate porosity (typically up to 10\%) to allow intergranular fluid flow through sandstones and export of diagenetic alteration products.

The compositions of the grain coatings measured here are unexceptional, because the sandstones did not have a known provenance in preexisting metalliferous ore deposits. If the sand and/or grain coating was derived from a metalrich source, red beds could be preloaded with metal that could more readily be leached to form an ore deposit. This is most likely where sand was eroded from an orogenic belt, in which metalliferous phases had been unroofed following 


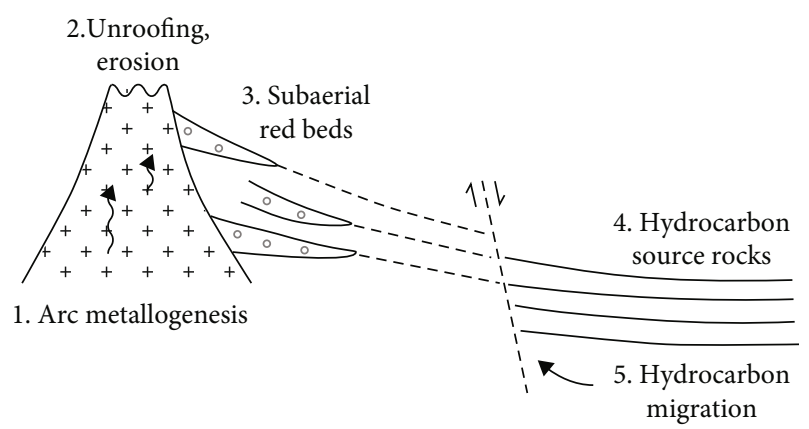

Figure 8: Schematic evolution of red bed ore deposit by erosion of magmatic arc rocks into red beds, followed by hydrocarbon migration through red beds to concentrate metals from metalbearing grain coatings.

uplift. In this way, arc-based deposits such as Cu porphyries, V-rich magnetite, and Se-rich volcanic soils could be recycled into sandstone ore feedstock. A mineralized plate margin mountain chain with subaerial erosion products (red beds) and a foreland basin (potential hydrocarbon source rocks) could provide a scenario where hydrocarbons strip enriched grain coatings from the red beds to create an ore fluid (Figure 8). For example, in the Tethyan region (Iran, Iraq, and adjacent areas), where red bed-hosted copper deposits are widespread [15], the Zagros and other orogenic mountain chains are fringed by red beds and hydrocarbon sources. In the Andes, copper mineralization in red beds has been attributed to the unroofing of arc volcanics [54]. Examples like these could be tested by mass balance studies of metals in source regions and ore deposits. In particular, the alteration of titanomagnetite is a suggested source of metals in red bed deposits [55], and there is evidence of such alteration in mineralized red beds $[54,56]$ which could be quantified. In the Andes, the magnetite in volcanic rocks is rich in vanadium [57], altered magnetite in the volcanic rocks shows the release of vanadium [58], and red beds are rich in magnetite grains [59], suggesting that this region would make a good case study. While the vanadium in titanomagnetite ores can be processed and reprecipitated by microbes in the soil zone (e.g., [60, $61]$ ), the contribution of microbial activity in the whole mineralization cycle has yet to be evaluated.

4.3. Limitations to Leaching Model. This study focused on red sandstones, as sandstones are potential aquifers for fluid flow and diagenetic reactions including dissolution of grain coatings. Red mudstones may contain higher contents of iron oxides and associated trace metals [50], as shown by the data from different grain size fractions at Gardenstown. Some trace metals in mudstones would be released into compactional brines, but they are not commonly pathways for the hydrocarbon migration envisaged to leach iron oxides from red sandstones. An exception is in mudstones which show evidence for overpressuring, which allows the flow of brines and hydrocarbons along bedding planes, a circumstance that may be promoted by impermeable evaporite beds that occur in some red bed sequences.
Leaching data reported by [8] are based largely on very young mudrocks of Holocene-Pliocene age in which the iron oxide crystallinity was not fully developed and allowed element liberation more readily than from fully crystalline oxides. Their data are nonetheless interesting in indicating a relative ease of leaching in which $\mathrm{Co}>\mathrm{Cu}>\mathrm{Fe}>\mathrm{U}>\mathrm{V}$. The relatively high availability of $\mathrm{Cu}$ recorded in the present study and by [8] may partially reflect the high adsorption (log $\mathrm{K}$ values) of copper onto iron oxides [62, 63]. $\mathrm{V}$ and $\mathrm{U}$ (and Se) might be harder to desorb if initially adsorbed as oxyanions [63]. A calculation based on leaching data for zinc in the red mudrocks concluded a volume of $6.10^{4} \mathrm{~km}^{3}$ rock was required to source a sedex deposit $[8,64]$, at least an order of magnitude more than the volume required to source a large copper deposit. The contrast in volumes required may reflect differential adsorption of the elements onto iron oxide $[62,63]$, whereby copper outcompetes zinc for adsorption, and consequently, desorption occurs at greater rates and possibly under different $\mathrm{pH}$ conditions.

Bleached sandstones at Alderley Edge locally contain secondary $\mathrm{Cu}$ minerals, indicating that leached metals are not necessarily exported from the system but may be reprecipitated by variable redox conditions. This is borne out by the high $\mathrm{Cu}$ contents in the bleached samples at Alderley Edge. This difference in mobility may also contribute to the poor correlation of $\mathrm{Cu}$ and $\mathrm{Fe}$ contents. Copper accumulation in bleached facies has similarly been observed in the Kupferschiefer [65], and it is evident as metal-enriched cores in reduction spheroids in many red beds [42]. The potential for leaching to create an oreforming fluid clearly requires a sedimentary basin scale and fluid flow system that are large enough to allow continued, cumulative transport of metals.

\section{Conclusions}

Measurement of trace elements resident in iron oxide grain coatings in red sandstones confirmed that they are a source of metals during diagenetic dissolution. In particular

(i) They represent a significant proportion of the metals available from the sandstones to ore-forming fluids

(ii) The quantities of metal in the grain coatings are adequate to form sandstone-hosted ore bodies. Only small proportions of available metal would be required to form $\mathrm{U}, \mathrm{V}$, and Co deposits, but the available $\mathrm{Cu}$ is still adequate to form moderate-size ore bodies if the process is efficient

(iii) A broad correlation between Fe content and the content of most trace metals confirms the predominant residence of the trace elements in the grain coatings, and hence, their availability to ore fluids

Future research should explore basins where red sandstones have a provenance which includes anomalous metal concentrations, for example, sandstones containing erosion products of mineralized granites or arc volcanics. 


\section{Data Availability}

The manuscript is a data self-contained article, whose results were obtained from the laboratory analysis, and the entire data are presented within the article. However, if any additional information is required, these are available from the corresponding author upon request to the e-mail j.parnell@abdn.ac.uk.

\section{Conflicts of Interest}

The authors declare that they have no known competing financial interests or personal relationships that could have appeared to influence the work reported in this paper.

\section{Acknowledgments}

The paper benefitted from constructive reviews by anonymous referees, for which we are very grateful. Sandstone samples were kindly contributed by the Geological Survey of Northern Ireland, D. Halbert, R. Starkey, and L. Bullock. This work was partly supported by NERC grants NE/M010953/1 and NE/T003677/1. Electron Microscopy was performed with the help of J. Still in the ACEMAC Facility at the University of Aberdeen. We thank TeledyneCETAC for the loan of the laser ablation system LSX-213 and support during the measurements.

\section{Supplementary Materials}

Table S1: compositions of grain coatings, with standard deviations, and data for certified reference materials. (Supplementary Materials)

\section{References}

[1] I. McDonald, A. J. Boyce, and I. B. Butler, Mineral Deposits and Earth Evolution, Geological Society of London, 2005.

[2] T. R. Walker, B. Waugh, and A. J. Crone, "Diagenesis in firstcycle desert alluvium of Cenozoic age, southwestern United States and northwestern Mexico," Geological Society of America Bulletin, vol. 89, no. 1, pp. 19-32, 1978.

[3] M. M. Benjamin, R. S. Sletten, R. P. Bailey, and T. Bennett, "Sorption and filtration of metals using iron-oxide-coated sand," Water Research, vol. 30, no. 11, pp. 2609-2620, 1996.

[4] R. C. Surdam, Z. S. Jiao, and D. B. MacGowan, "Redox reactions involving hydrocarbons and mineral oxidants: a mechanism for significant porosity enhancement in sandstones," AAPG Bulletin, vol. 77, pp. 1509-1518, 1993.

[5] W. T. Parry, M. A. Chan, and B. P. Nash, "Diagenetic characteristics of the Jurassic Navajo sandstone in the covenant oil field, Central Utah thrust belt," American Association of Petroleum Geologists Bulletin, vol. 93, no. 8, pp. 1039-1061, 2009.

[6] M. Wigley, N. Kampman, B. Dubacq, and M. Bickle, "Fluidmineral reactions and trace metal mobilization in an exhumed natural CO2 reservoir, Green River, Utah," Geology, vol. 40, no. 6, pp. 555-558, 2012.

[7] M. Wigley, N. Kampman, H. J. Chapman, B. Dubacq, and M. J. Bickle, "In situ redeposition of trace metals mobilized by CO2charged brines," Geochemistry Geophysics Geosystems, vol. 14, no. 5, pp. 1321-1332, 2013.
[8] R. A. Zielinkski, S. Bloch, and T. R. Walker, "The mobility and distribution of heavy metals during the formation of first cycle red beds," Economic Geology, vol. 78, no. 8, pp. 1574-1589, 1983.

[9] T. R. Walker, "Application of diagenetic alterations in red beds to the origin of copper in stratiform copper deposits," Geological Association of Canada Special Paper, vol. 36, pp. 85-96, 1989.

[10] A. W. Rose and G. C. Bianchi-Mosquera, "Adsorption of $\mathrm{Cu}$, $\mathrm{Pb}, \mathrm{Zn}, \mathrm{Co}, \mathrm{Ni}$, and $\mathrm{Ag}$ on goethite and hematite: a control on metal mobilization from red beds into stratiform copper deposits," Economic Geology, vol. 88, no. 5, pp. 1226-1236, 1993.

[11] R. Metcalfe, C. A. Rochelle, D. Savage, and J. W. Higgo, "Fluidrock interactions during continental red bed diagenesis: implications for theoretical models of mineralization in sedimentary basins," Geological Society, London, Special Publications, vol. 78, no. 1, pp. 301-324, 1994.

[12] A. C. Brown, "A process-based approach to estimating the copper derived from red beds in the sediment-hosted stratiform copper deposit model," Economic Geology, vol. 104, no. 6, pp. 857-868, 2009.

[13] P. M. Cossette, A. A. Bookstrom, T. S. Hayes, G. R. Robinson, J. C. Wallis, and M. L. Zientek, Sandstone Copper Assessment of the Teniz Basin, Kazakhstan, United States Geological Survey Scientific Investigations Report, 2010.

[14] S. E. Box, B. Syusyura, R. Seltmann, R. A. Creaser, A. Dolgopolova, and M. L. Zientek, "Dzhezkazgan and associated sandstone copper deposits of the Chu-Sarysa Basin, Central Kazakhstan," Society of Economic Geologists Special Publication, vol. 16, pp. 303-328, 2012.

[15] S. Maghfouri, E. Rastad, G. Borg et al., "Metallogeny and temporal-spatial distribution of sediment-hosted stratabound copper (SSC-type) deposits in Iran; implications for future exploration,” Ore Geology Reviews, vol. 127, p. 103834, 2020.

[16] S. Jaireth, A. McKay, and I. Lambert, "Association of large sandstone uranium deposits with hydrocarbons," AusGeo News, vol. 89, no. 6, pp. 1-6, 2008.

[17] A. L. Rainoldi, M. Franchini, D. Beaufort et al., "Large-scale bleaching of red beds related to upward migration of hydrocarbons: Los Chihuidos High, Neuquen Basin, Argentina," Journal of Sedimentary Research, vol. 84, no. 5, pp. 373-393, 2014.

[18] A. L. Rainoldi, M. B. Franchini, A. J. Boyce et al., "Stable isotope and fluid inclusion study of sediment-hosted stratiform copper deposits from the Neuquén Basin, Argentina," Mineralium Deposita, vol. 54, no. 3, pp. 415-436, 2019.

[19] A. L. Rainoldi, D. Beaufort, P. Patrier, A. Giusiano, M. J. Pons, and N. Cesaretti, "Vanadium mineralization at Los Chihuidos sediment-hosted stratiform Cu deposit," in Proceedings of the 15th Quadrennial IAGOD Symposium, pp. 355-356, Salta, Argentina, 2018.

[20] J. Mathieu, E. C. Turner, D. J. Kontak, M. Fayek, and R. Mathur, "Atypical $\mathrm{Cu}$ mineralisation in the Cornwallis carbonate-hosted $\mathrm{Zn}$ district: storm copper deposit, Arctic Canada," Ore Geology Reviews, vol. 99, pp. 86-115, 2018.

[21] J. G. Hinchey, "Neoproterozoic sedimentary-hosted 'stratiform' copper mineralization - Bonavista Peninsula, Avalon Zone, Newfoundland: initial field and petrographic observations," Current Research. Newfoundland and Labrador Department of Natural Resources, Geological Survey, vol. 2010, pp. 121, 2010. 
[22] R. Gao, C. Xue, X. Zhao, X. Chen, Z. Li, and D. Symons, "Source and possible leaching process of ore metals in the Uragen sandstone- hosted $\mathrm{Zn}-\mathrm{Pb}$ deposit, Xinjiang, China: Constraints from lead isotopes and rare earth elements geochemistry," Ore Geology Reviews, vol. 106, pp. 56-78, 2019.

[23] B. Wu, G. Zheng, A. Zhang et al., "The different characteristics and geological significance of reduction alteration of sandstone-type uranium deposits in northwest region of China," Energy Exploration \& Exploitation, vol. 27, no. 4, pp. 261-275, 2009.

[24] H. Chu, G. Chi, S. Bosman, and C. Card, "Diagenetic and geochemical studies of sandstones from drill core DV10-001 in the Athabasca basin, Canada, and implications for uranium mineralization," Journal of Geochemical Exploration, vol. 148, pp. 206-230, 2015.

[25] P. Turner, Continental Red Beds, Elsevier, Amsterdam, UK, 1980.

[26] J. Parnell, S. Spinks, and C. Brolly, "Tellurium and selenium in Mesoproterozoic red beds," Precambrian Research, vol. 305, pp. 145-150, 2018.

[27] W. H. Wang, "Origin of reddening and secondary porosity in Carboniferous sandstones, Northern Ireland," Geological Society Special Publication, vol. 62, no. 1, pp. 243-254, 1992.

[28] D. Richter, "On the new red sandstone neptunian dykes of the Tor Bay Area (Devonshire)," Proceedings of the Geologists Association, vol. 77, no. 2, pp. 173-IN3, 1966.

[29] R. A. Ixer and D. J. Vaughan, "The primary ore mineralogy of the Alderley Edge deposit, Cheshire," Mineralogical Magazine, vol. 46, no. 341, pp. 485-492, 1982.

[30] J. Rowe and S. D. Burley, "Faulting and porosity modification in the Sherwood Sandstone at Alderley Edge, northeastern Cheshire: an exhumed example of fault-related diagenesis," Geological Society Special Publication, vol. 124, no. 1, pp. 325-352, 1997.

[31] J.-H. Choi, J.-S. Ryu, H. S. Shin, J. Kim, and C.-s. Cheong, "Reevaluation of Th and $\mathrm{U}$ concentrations in marine sediment reference materials using isotope dilution MC-ICP-MS: towards the analytical improvements in dose rate estimation for luminescence dating," Geosciences Journal, vol. 17, no. 2, pp. 123-127, 2013.

[32] H. L. Byers, L. J. McHenry, and T. J. Grundl, "Forty-nine major and trace element concentrations measured in soil reference materials NIST SRM 2586, 2587, 2709a, 2710a and 2711a using ICP-MS and wavelength dispersive-XRF," Geostandards and Geoanalytical Research, vol. 40, pp. 443-455, 2016.

[33] M. R. Cave and K. Harmon, "Determination of trace metal distributions in the iron oxide phases of red bed sandstones by chemometric analysis of whole rock and selective leachate data," Analyst, vol. 122, no. 6, pp. 501-512, 1997.

[34] S. R. Huelin, H. P. Longerich, D. H. C. Wilton, and B. J. Fryer, "The determination of trace elements in Fe-Mn oxide coatings on pebbles using LA-ICP-MS," Journal of Geochemical Exploration, vol. 91, no. 1-3, pp. 110-124, 2006.

[35] A. Schmidt Mumm, R. C. Dart, and P. Say, "Hematite/maghemite trace element geochemistry in base metal exploration," Journal of Geochemical Exploration, vol. 124, pp. 239-251, 2013.

[36] M. B. Goldhaber, R. L. Reynolds, J. A. Campbell, R. B. Wanty, R. I. Grauch, and R. Northrop, "Mechanism of ore and gangue mineral formation at the interface between brine and meteoric water," Economic Geology, vol. 85, pp. 236-250, 1990.
[37] W. R. Fischer and U. Schwertmann, "The formation of hematite from amorphous iron (III) hydroxide," Clays and Clay Minerals, vol. 23, no. 1, pp. 33-37, 1975.

[38] A. J. Frierdich, Y. Luo, and J. G. Catalano, "Trace element cycling through iron oxide minerals during redox-driven dynamic recrystallization," Geology, vol. 39, no. 11, pp. 10831086, 2011.

[39] T. Schirmer, A. Koschinsky, and M. Bau, "The ratio of tellurium and selenium in geological material as a possible paleoredox proxy," Chemical Geology, vol. 376, pp. 44-51, 2014.

[40] J. P. Gustafsson, "Vanadium geochemistry in the biogeosphere - speciation, solid-solution interactions, and ecotoxicity," Applied Geochemistry, vol. 102, pp. 1-25, 2019.

[41] M. Van Panhuys-Sigler, N. H. Trewin, and J. Still, "Roscoelite associated with reduction spots in Devonian red beds, Gamrie Bay, Banffshire," Scottish Journal of Geology, vol. 32, no. 2, pp. 127-132, 1996.

[42] J. Parnell, S. Spinks, and D. Bellis, "Low-temperature concentration of tellurium and gold in continental red bed successions," Terra Nova, vol. 28, no. 3, pp. 221-227, 2016.

[43] A. J. Urban, B. F. Hoskins, and I. E. Grey, "Characterization of $\mathrm{V}-\mathrm{Sb}-\mathrm{W}$-bearing rutile from the Hemlo gold deposit, Ontario," Canadian Mineralogist, vol. 30, pp. 319-326, 1992.

[44] A. V. Boitsov, "The mineral composition and the ore types of the uranium-vanadium deposit Srednaya Padma (Onega Region, Russian Federation)," in Technical Committee Meeting on Changes and Events in Uranium Deposit Development, Exploration, Resources, Production and the World SupplyDemand Relationship, pp. 259-269, IANA, Veinna, Austria, 1997.

[45] D. J. Noy, S. Holloway, R. A. Chadwick, J. D. O. Williams, S. A. Hannis, and R. W. Lahann, "Modelling large-scale carbon dioxide injection into the Bunter Sandstone in the UK Southern North Sea," International Journal of Greenhouse Gas Control, vol. 9, pp. 220-233, 2012.

[46] J. D. O. Williams, M. Bentham, M. Jin et al., "The effect of geological structure and heterogeneity on $\mathrm{CO} 2$ storage in simple 4-way dip structures; a modeling study from the UK Southern North Sea," Energy Procedia, vol. 37, pp. 3980-3988, 2013.

[47] S. Thibeau, S. Bachu, J. Birkholzer, S. Holloway, F. Neele, and Q. Zhou, "Using pressure and volumetric approaches to estimate CO2 storage capacity in deep saline aquifers," Energy Procedia, vol. 63, pp. 5294-5304, 2014.

[48] D. L. Mosier, D. A. Singer, and D. P. Cox, "Grade and tonnage model of sediment-hosted Cu," in Mineral Deposit Models, D. P. Cox and D. A. Singer, Eds., vol. 1693, pp. 206-208, U.S. Geological Survey Bulletin, 1992.

[49] I. Holmes, A. D. Chambers, R. D. Ixer, P. Turner, and D. J. Vaughan, "Diagenetic processes and the mineralization in the Triassic of Central England," Mineralium Deposita, vol. 18, pp. 365-377, 1983.

[50] J. A. Plant, D. G. Jones, and H. W. Haslam, The Cheshire Basin - Basin Evolution, Fluid Movement and Mineral Resources in a Permo-Triassic Rift Setting, British Geological Survey, Keyworth, UK, 2000.

[51] S. Thakur, B. Chudasama, and A. Porwal, "Global grade-andtonnage modeling of uranium deposits," in Quantitative and Spatial Evaluations of Undiscovered Uranium Resources (IAEA-TECDOC-1861), pp. 226-272, International Atomic Energy Agency, Vienna, Austria, 2018. 
[52] K. D. Kelley, C. T. Scott, D. E. Polyak, and B. E. Kimball, "Vanadium," in Critical Mineral Resources of the United States - Economic and Environmental Geology and Prospects for Future Supply, K. J. Schulz, J. H. DeYoung, R. R. Seal, and D. C. Bradley, Eds., pp. U1-U36, U.S. Geological Survey Professional Paper, 2017.

[53] J. F. Slack, B. E. Kimball, and K. B. Shedd, Cobalt, United State Geological Survey Professional Paper, 2017.

[54] S. Flint, H. Clemmey, and P. Turner, "Conglomerate-hosted copper mineralization in cretaceous Andean molasse: the Coloso Formation of northern Chile," Geological Magazine, vol. 123, no. 5, pp. 525-536, 1986.

[55] R. L. Reynolds and M. B. Goldhaber, "Origin of a South Texas roll-type uranium deposit: I. Alteration of iron-titanium oxide minerals," Economic Geology, vol. 73, no. 8, pp. 1677-1689, 1978.

[56] R. Weibel and H. Friis, "Opaque minerals as keys for distinguishing oxidising and reducing diagenetic conditions in the Lower Triassic Bunter Sandstone, North German Basin," Sedimentary Geology, vol. 169, no. 3-4, pp. 129-149, 2004.

[57] S. A. S. Dare, S.-J. Barnes, G. Beaudoin, J. Méric, E. Boutroy, and C. Potvin-Doucet, "Trace elements in magnetite as petrogenetic indicators," Mineralium Deposita, vol. 49, no. 7, pp. 785-796, 2014.

[58] K. Uchida, "Minor elements of magnetite in unaltered and altered host rocks of Michiquillay porphyry copper deposit," Peru. Mining Geology, vol. 26, pp. 153-163, 1976.

[59] P. Roperch, C. Arriagada, A. Chauvin, and V. Carlotto, Tectonic Imprint in Magnetic Fabrics in Sediments from the Central Andes, AGU Spring Meeting Abstracts, 2009.

[60] L. Hao, B. Zhang, C. Feng et al., "Microbial vanadium (V) reduction in groundwater with different soils from vanadium ore mining areas," Chemosphere, vol. 202, pp. 272-279, 2018.

[61] B. Zhang, S. Wang, M. Diao et al., "Microbial community responses to vanadium distributions in mining geological environments and bioremediation assessment," Journal of Geophysical Research: Biogeosciences, vol. 124, no. 3, pp. 601615, 2019.

[62] D. A. Dzombak and F. M. M. Morel, Surface Complexation Modeling: Hydrous Ferric Oxide, John Wiley \& Sons, New York, NY, USA, 1990.

[63] S. S. Mathur and D. A. Dzombak, "Surface complexation modeling: goethite," in Surface Complexation Modeling, J. Lützenkirchen, Ed., pp. 443-468, Elsevier, Amsterdam, UK, 2006.

[64] P. Emsbo, R. R. Seal, G. N. Breit, S. F. Diehl, and A. K. Shah, Sedimentary Exhalative (sedex) Zinc-Lead-Silver Deposit Model, pp. 1-57, U.S. Geological Survey, Scientific Investigations Report 2010-5070-N, 2016.

[65] S. Oszczepalski, "Origin of the Kupferschiefer polymetallic mineralization in Poland," Mineralium Deposita, vol. 34, no. 5-6, pp. 599-613, 1999. 